\title{
MAGNETO-OPTICAL STUDIES OF NOVEL EPITAXIAL FILMS
}

\author{
S. D. BADER and E. R. MOOG \\ Materials Science Division, Argonne National Laboratory, Argonne, IL 60439, USA
}

\section{ABSTRACT}

The magneto-optic Kerr-effect technique is used to explore the magnetic properties of ultrathin epitaxial films of iron and chromium grown on (100) faces of gold and copper. The studies extend into the monolayer range, and are performed in the ultra-high-vacuum environment in which the films are grown. Auxiliary low-energy electron diffraction and Auger electron spectroscopy studies are used to characterize the epitaxy and growth-mode behavior. Highlights include: (a) an observed lowering of the Curie temperature, compared to that of the bulk, for monolayer-range iron grown epitaxially on the lattice-matched (100) face of gold; (b) correlations between magnetism and morphology; (c) a search for the predicted ferromagnetism of chromium monolayers; and (d) the observation of a new, metastable surface magnetic state for the high-temperature, face-centeredcubic phase of Iron, which is stabilized via epitaxy on the (100) face of copper. The opportunities, challenges and limitations posed by the present magneto-optical approach in assessing the properties of these atomically-engineered materials will be evaluated.

\section{INTRODUCTION}

Recently the magneto-optic Kerr effect has proved to be valuable in the study of the magnetic properties of ultra-thin epitaxial films.[1,2] Interest in epitaxial metallic films has increased due to two technological developments. Firstly, advances in ultra-high-vacuum (UHV) techniques for high-quality film preparation and characterization have ushered in a new era with hopes of atomically engineering desired properties modifications.[3] Secondly, advances in supercomputer capabilities have permitted realistic modeling, and the hope of being able to make reliable predictions, within the local-density approximation. [4] Interest in the magnetic properties of these films centers on their use as model systems to probe two-dimensional magnetism, to explore the relationship between phase stability and magnetism, and to isolate the basic building block of the magnetic-superlattice interface. Examples are provided in the present work of the type of information simple magneto-optical experiments can provide when coupled to conventional electron spectroscopic and diffraction characterizations. The magnetic properties of Fe stabilized in the body-centered cubic (bcc) and face-centered cubic (fcc) phases, via epitaxy on Au(100) and $\mathrm{Cu}(100)$, respectively, are shown to be strikingly different. Also, relationships between morphology and magnetism are reflected in the shape of the magnetic hysteresis loop. Furthermore, a search for the predicted ferromagnetism of surface and interfacial Cr illustrates the dynamic interaction between current experimental and computational efforts.

\section{EXPERIMENTAL PROCEDURES}

\section{A. Epitaxy and Growth Mode}

The experiments are performed in a UHV chamber of base pressure in the $10^{-11}$ Torr range. Evaporators, as described elsewhere, are used to deposit $\mathrm{Fe}$ or $\mathrm{Cr}$ on $\mathrm{Au}(100)$ or $\mathrm{Cu}(100) .[1],[5]$ The bulk-terminated surfaces of these crystals are well lattice-matched for bcc and fcc growth, respectively. This might not at first be apparent for Au, which is an fcc metal; but its bulk lattice constant of $4.08 \AA$ yields a $(100)$-face primitive square-net lattice constant of $4.08 / \sqrt{2}=2.88 \AA$, which coincides with the bcc lattice constants of both $\mathrm{Cr}(2.88 \AA)$ and $\mathrm{Fe}$ $(2.87 \AA)$. Low-energy electron diffraction (LEED) verifies that the anticipated epitaxy has actualiy taken place. Somewhat elevated growth temperatures $\left(100-200^{\circ} \mathrm{C}\right)$ produce the sharpest LEED spots because enhanced surface diffusion promotes ordering. Further increase in substrate temperatures, however, tends to be undesirable, due to bulk-diffusion effects and alloying. Auger electron spectroscopy is used to monitor the surface cleanliness, the initfal growth mode, and the rate of evaporation. Growth rates of order 5 minutes per monolayer allow numerous samplings of the Auger spectrum at intervals during the growth process. Results are shown in Fig. 1 for $\mathrm{fcc}-\mathrm{Fe} / \mathrm{Cu}(100)$, where the rise of the Fe Auger signals and the attenuation of the Cu signals are shown as the deposition proceeds. The solid curves are the results of simple calculations performed for layer-by-layer growth models. The primary input to the calculation is the electron mean free path for the appropriate Auger electron energy; it is obtained from the universal curve known to all electron spectroscopists. The curves that most closely agree with the data assume somewhat imperfect layer-by-layer growth: the $(i+1)$ th layer is $15 \%$ complete by the time the $i^{\text {th }}$ layer growth 1s $100 \%$ complete. The other curve is for perfectly ideal layer-by-layer growth. The growth is classified as being essentially near-ideal layer-by-layer, based on the agreement in Fig. 1 between experiment and model calculations. The fcc-Fe/Cu(100) case provides a good example of the quality of film that can be produced in the laboratory. 


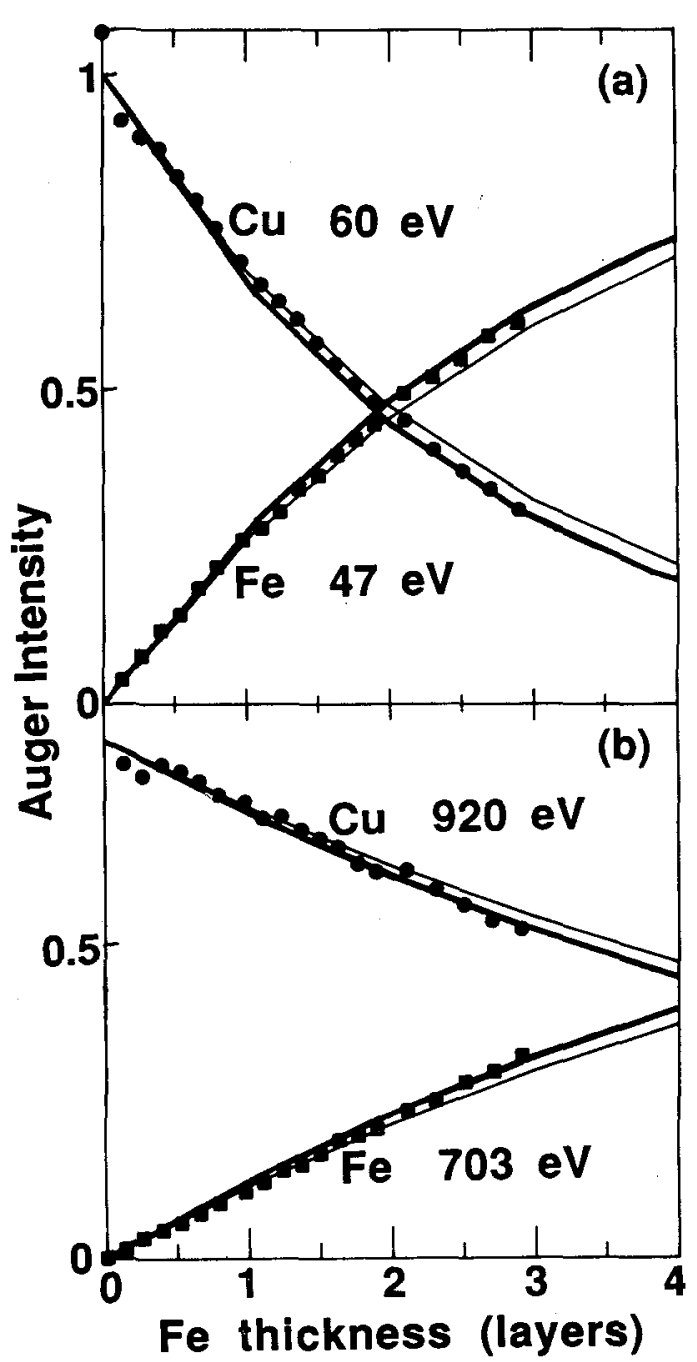

FIG. 1. Experimental and calculated Auger growth curves for $\mathrm{fcc}-\mathrm{Fe} / \mathrm{Cu}(100)$ at $150^{\circ} \mathrm{C}$, for the indicated Auger transition energies.

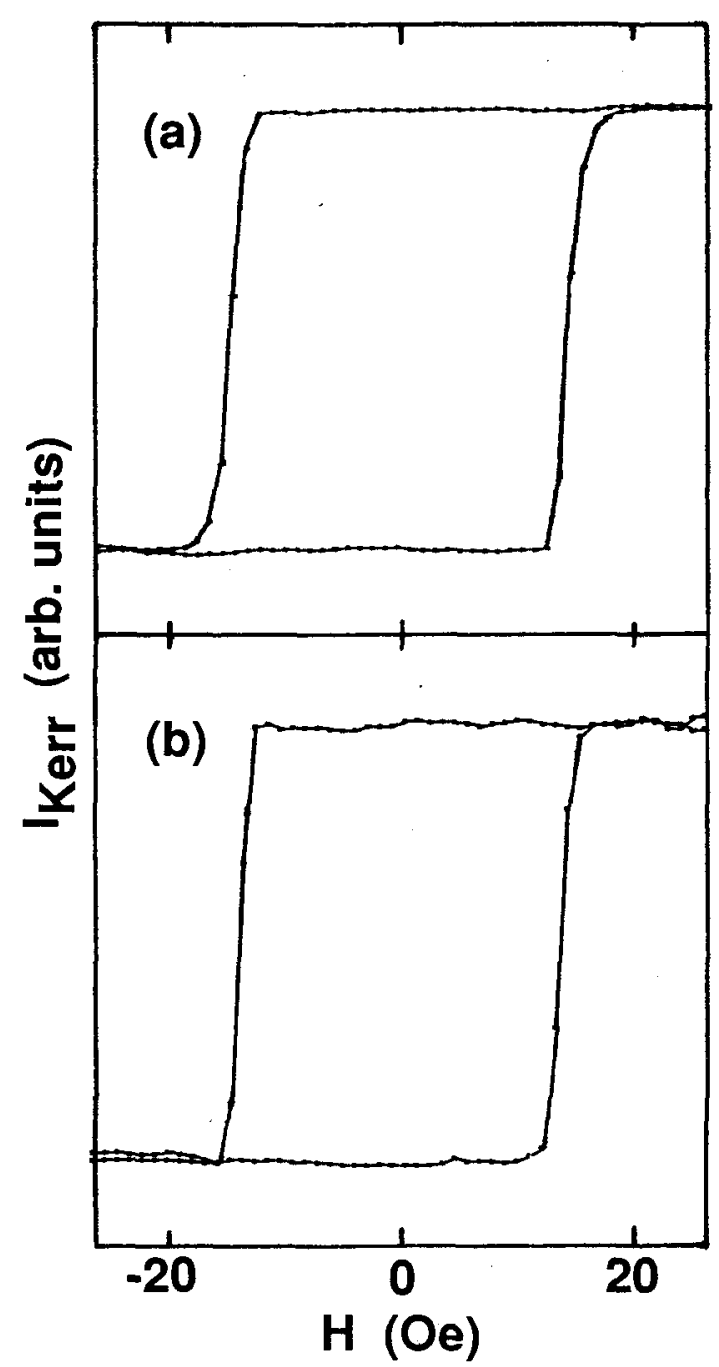

FIG. 2. Comparison of d.c. (pane1 a) and a.c. (pane1 b) Kerr-effect measurements on the same Fe film.

\section{B. Magneto-Optics}

The Kerr-effect experiment is performed in situ by shining a He-Ne laser through a UHV window at the magnetized sample. The reflected light passes through a crystal-prism polarizer to a photodiode detector. A laboratory computer controls the current to the in-situ electromagnet, and digitizes the detector output. The computer also signal averages multiple hysteresis-loop scans to improve signal to noise. The sample is magnetized along an in-plane [001] easy axis that, along with the incident-1ight polarization, lies in the scattering plane. This arrangement yields a Kerr intensity $I_{K}$ as the magnetic field is swept. No mechanical adjustments are made in the analyzer orientation or in the scattering angle. In Fig. 2 data are shown to compare this simple d.c. technique to an a.c. scheme that utilizes photoelastic modulation of the incident beam, and a lock-in amplifier on the photodiode detector output.[6] The experiment was performed in air on a thick iron film grown on sapphire and covered with an anti-reflection coating to enhance the signa1. In both panels of Fig. 2 the data collection time was $\sim 30$ sec. The d.c. signal in panel 2(a), a computer average of 5 scans, somewhat surpasses in quality that in panel 2(b), a single a.c. scan with phase-sensitive detection. The modulator and lock-in amplifier were, thus, not used in the experiments that follow. The only additional optical element, used occasionally, was a Solei1-Babinet compensator, which corrected for the birefringence of the UHV window.

\section{RESULTS AND DISCUSSION}

\section{A. $\mathrm{Fe} / \mathrm{Au}(100)$}

Rerr Intensity data for the system $\mathrm{Fe} / \mathrm{Au}(100)$ are summarized in Figs. 3 and 4 as a function of applied magnetic field $H$ and temperature $T$. The hysteresis loops in Fig. 3 were collected in a few minutes time. Differences in the data quality between Fig. 3 and Fig. 2(a) are attributed to the films of Fig. 3 being ultra-thin and devoid of coating, and there being a UHV window between the sample and measurement equipment in Fig. 3 and not in $2(\mathrm{a})$. Nonetheless, the squareness of the loops in Fig. 3 attests to the quality of the films. The changes with temperature in the loop characteristics between Fig. $3(b)$ and (c) are not representative of bulk iron, but are indicative of the expected reduction in Curie temperature $T_{C}$ for monolayer-range films. This point is illustrated further in Fig. 4, where the normalized Kerr intensity in the remanent state and the coer- 

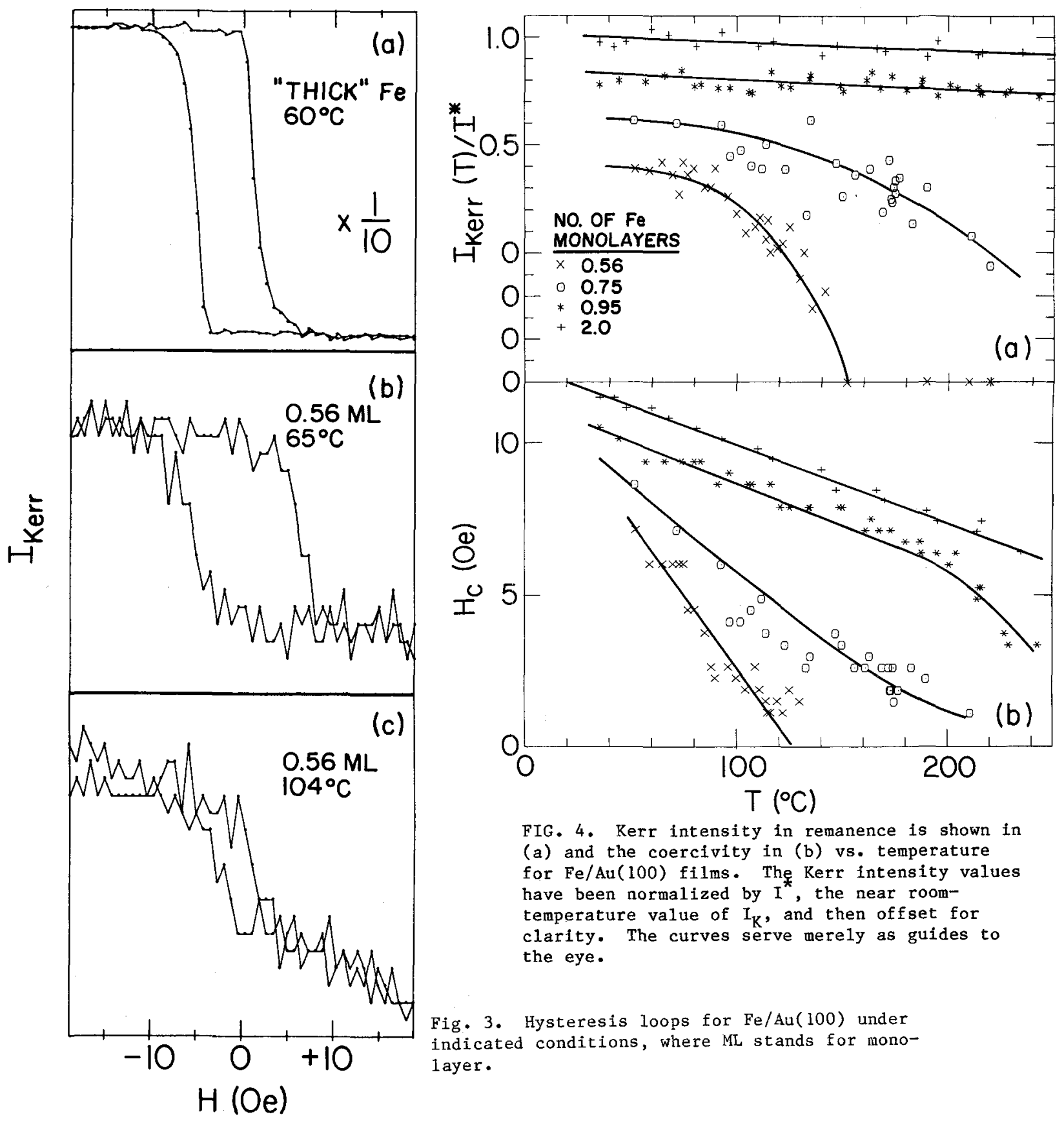

FIG. 4. Kerr intensity in remanence is shown in (a) and the coercivity in (b) vs. temperature for $\mathrm{Fe} / \mathrm{Au}(100)$ films. The Kerr intensity values have been normalized by $I^{*}$, the near roomtemperature value of $I_{K}$, and then of $f$ set for clarity. The curves serve merely as guides to the eye.

Fig. 3. Hysteresis loops for $\mathrm{Fe} / \mathrm{Au}(100)$ under indicated conditions, where ML stands for monolayer.

civity $H_{\text {c }}$ are plotted vs. T in panels (a) and (b), respectively, for the indicated monolayer-range Fe dosages. The data were taken on both heating and cooling cycles, to ensure reproducibility, but do not extend to higher temperatures because of the onset of bulk-diffusion effects.

A trend can be discerned, especially in the $\mathrm{H}$ data of Fig. 4(b), of $\mathrm{T}_{\mathrm{C}}$ increasing as the $\mathrm{Fe}$ dosage increases. The lowest-dosage film of Fig. 4 is observed to have a $\mathrm{T}_{\mathrm{C}}^{\mathrm{C}}$ value of $\sim 150^{\circ} \mathrm{C}$, which is $\sim 40 \%$ of the bulk $T_{C}$ value of iron. This is in rough accord with simple mean-fieldtheory expectations based on the number of nearest neighbors at the surface being half that in the bulk. [7] Monolayer-range ferromagnetism at such high temperatures is a consequence of strong surface anisotropy. This is in stark contrast, for example, to the zero-temperature-ordering prediction of the two-dinensional isotropic Heisenberg model.[8] It has recently been suggested that the anisotropy of nonolayer-range Fe films should cause the spins to be oriented perpendicular to the film plane.[9],[10] However, the present $\mathrm{Fe} / \mathrm{Au}(100)$ results do not support this 1dea. See Ref. 11 for more complete characterizations and discussion of the $\mathrm{Fe} / \mathrm{Au}(100)$ system.

\section{B. Magnetism and Morphology}

Examples are presented in this subsection of how the character of the hysteresis loop provides auxiliary information on the morphology and initial growth mechanism of ultra-thin films. Three classical growth modes are the:

a) layer-by-layer, or Frank-van der Merwe (FvdM),

b) 1ayer-plus-island, or Stranski-Krastanov (SK), and

c) island, or Volmer-Weber (VW), growth modes.

The distinctive ferromagnetic hysteresis loops of the previous subsection tend to rule out VW 


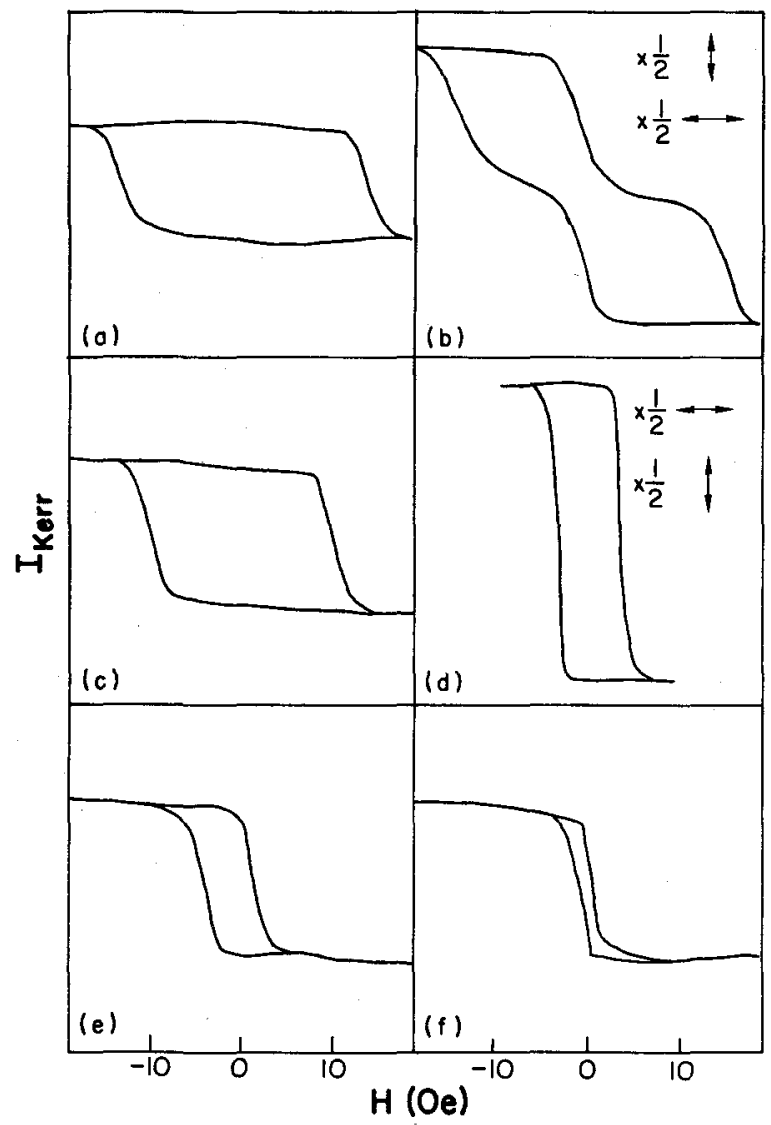

FIG. 5. Hysteresis loops for films grown in different ways, resulting in different morphologies. Panels (a) and (b) show the loops for 1 and 5 layers, respectively, of $\mathrm{Fe} / \mathrm{Au}(100)$ grown near room temperature. Panels (c) and (d) show corresponding loops for films grown at $230^{\circ} \mathrm{C}$. Panel (e) is for 1 layer of $\mathrm{Fe}$ on $\mathrm{Au}(100)$ with $1 / 4$ layer of $\mathrm{Cr}$ deposited subsequently. Panel ( $f$ ) is for 2 layers of $\mathrm{Cr}$ on $\mathrm{Au}(100)$ with a layer of Fe deposited subsequently. The loops have been smoothed by hand to eliminate the distracting noise pattern, as observed in Fig. 3.

growth because islanding is expected to be associated with superparamagnetic, not ferromagnetic, behavior. Evidence presented in Fig. 5(a)-(d) suggests that when the substrate is held at room temperature SR growth predominates, while at elevated temperature $\left(\sim 100-200^{\circ} \mathrm{C}\right)$ FvdM growth predominates. This is inferred because one monolayer (ML) Fe deposition gives similar square loops for either growth temperature [see Fig. 5(a) and (c)], while 5ML Fe deposition yields a qualitatively different loop for room-temperature growth. The loop in Fig. 5(b) has a butterfly-type shape characteristic of two-phase material, identified here as the smooth and rough phases, corresponding to layered and islanded regions, respectively. The islands on top of the smooth layer introduce a shape anisotropy that tends to stabilize differently oriented easy axes and, thus, to destroy the remanent state. This explanation is supported by the LEED observation presented in Sec. $2 \mathrm{~A}$ that elevated-temperature growth yields sharper LEED beams than room-temperature growth.

A final example will anticipate some of the findings of the next subsection on the search for ferromagnetism of $\mathrm{Cr}$. In this exercise $\mathrm{Cr}$ was deposited on $1 \mathrm{ML} \mathrm{Fe}$ on $\mathrm{Au}(100)$, and 1 ML $\mathrm{Fe}$ was deposited on $\mathrm{Cr}$ on $\mathrm{Au}(100)$, as is shown in $\mathrm{Fig} .5(\mathrm{e})$ and $(\mathrm{f})$ respectively. If the Fe were to Induce the $\mathrm{Cr}$ to order ferromagnetically, the height of the hysteresis curve, which is proportional to the magnetization, would change. If the $\mathrm{Cr}$ were to couple ferromagnetically to the $\mathrm{Fe}$ layer the signal would grow larger than that for the $F e$ alone; if the $C r$ were to couple antiferromagnetically, the signal would become smaller.[12] Comparison of the signal levels in Fig. 5(e) and $(\mathrm{f})$ to those of $\mathrm{Fig} .5(\mathrm{a})$ or (c) shows that neither anticipated result occurs. The magnetization remains that of the original Fe film. However, the coercivity decreases substantially in both cases from that of the Fe film. The $H_{c}$ values here become suggestive of magnetic-glass behavior, where the crystalline anisotropy is destroyed due to disorder. Indeed, the LEED patterns are totally disordered, and it is suspected that the two transition metals have intermixed.

\section{Search for Ferromagnetic Cr Monolayers}

Density-functional calculations have shown that both the surface of $\mathrm{Cr}(100)$ and monolayer $\mathrm{Cr}$ grown epitaxially on Au(100) may exhibit ferromagnetism with greatly enhanced magnetic moments of up to $3.6 \mu_{B}$ compared to the bulk antiferromagnetic value of $0.59 \mu_{B} \cdot[4],[12],[13]$ Experimentally the status of the $\operatorname{Cr}(100)$ surface has been controversial and unresolved.[14]-[18], [5] In the present study a ferromagnetic magneto-optic response was sought unsuccessfully for $T \geqslant 100 \mathrm{~K}$ for a variety of monolayer-range dosages of $\mathrm{Cr}$ grown epitaxially on $\mathrm{Au}(100)$. This null result could be attributed to many effects other than a lack of ferromagnetism: for instance, the $T_{C}$ value could be below $100 \mathrm{~K}$, or a $\mathrm{high}-\mathrm{H}$ value could preclude the film being magnetized by the weak applied fields. However, a considerably higher $T_{C}$ value is expected, at least for the surface of $\operatorname{Cr}(100)$, based both on numerical estimates of Grempel, [19] and experimental inferences of Klebanoff, et a1.[16] Also, the $\mathrm{Cr}-\mathrm{Fe}$ experiments of the previous subsection were originally performed in an attempt to overcome the limitation of the weak applied field. Those experiments also failed to provide evidence that the $\mathrm{Cr}$ possesses an ordered moment. In any case, motivated by the present work, Freeman's group subsequently calculated that an antiferromagnetic spin alignment within the film plane yielded an even lower total energy than for either the paramagnetic or ferromagnetic cases considered earlier.[20] This provides both a new explanation for the lack of experimental 


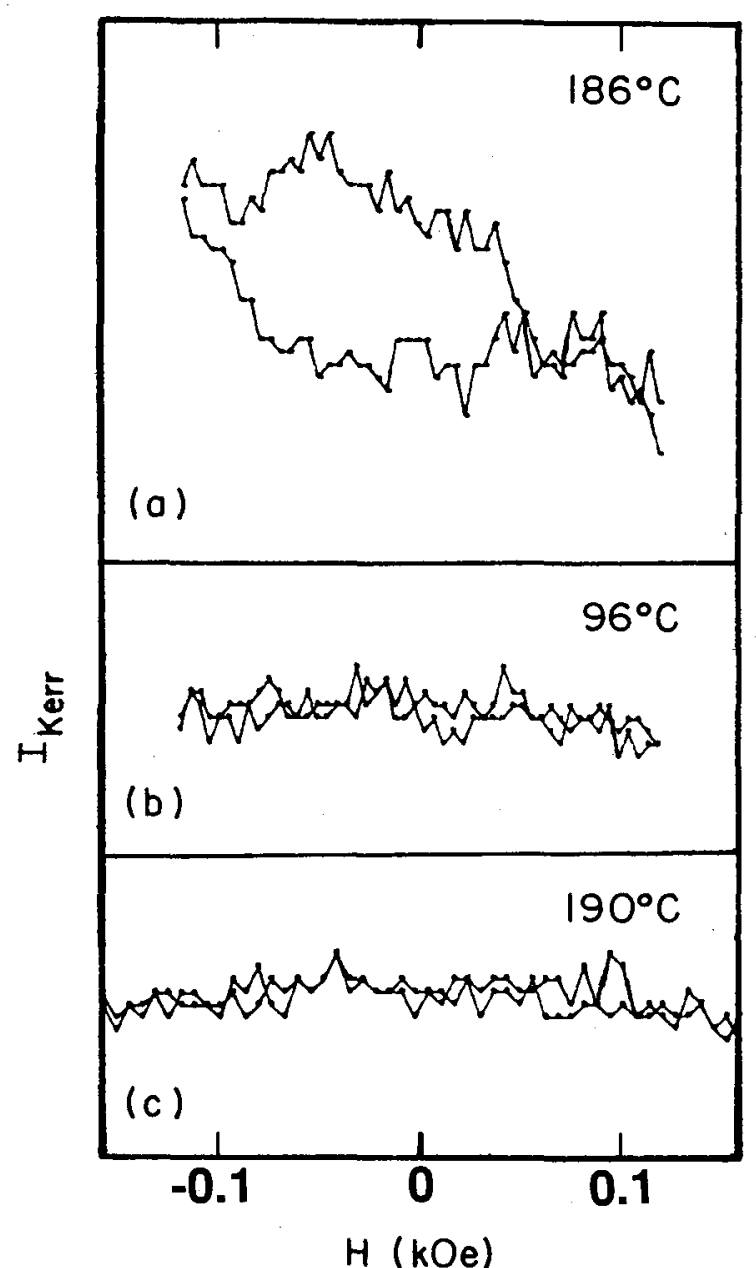

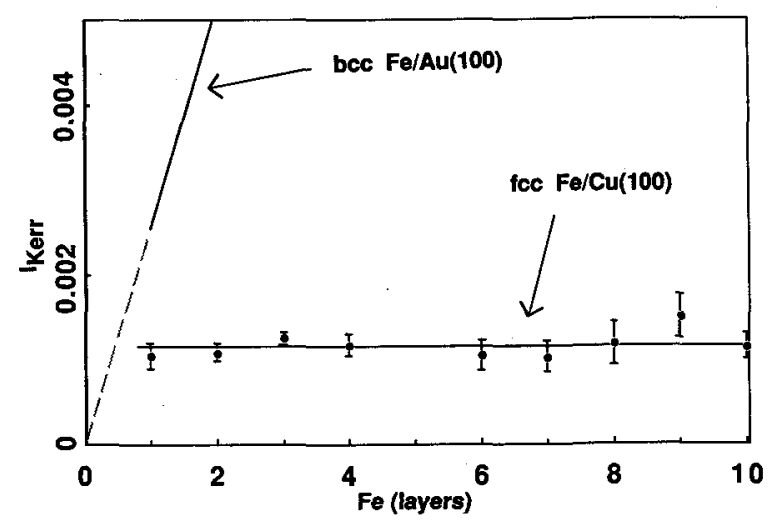

FIG. 7. Remanent Kerr intensity, normalized by total reflected intensity, as a function of $\mathrm{Fe}-$ film thickness. The $\mathrm{fcc}-\mathrm{Fe} / \mathrm{Cu}(100)$ data points are averaged values obtained from 34 measurements of 12 different films with r.m.s. deviation error bars attached; the line through the data is the average measured value.

FIG. 6. A ferromagnetic hysteresis loop is shown in (a) for 3 layers of $\mathrm{fcc}-\mathrm{Fe} / \mathrm{Cu}(100)$ grown and measured at $\sim 190^{\circ} \mathrm{C}$. Panels (b) and (c) show that the signal disappears on cooling and does not reappear upon reheating.

ferromagnetic response, and also an excellent example of how the interplay of experimental and computational physics can broaden our insights into challenging systems of interest.

\section{Face-Centered Cubic $\mathrm{Fe} / \mathrm{Cu}(100)$}

There has been much theoretical interest in the extreme volume dependence of the magnetic properties of $\mathrm{fcc}$ or $\gamma-\mathrm{Fe} .[21]$ Para-, ferro-, and antiferromagnetic ground states have been predicted at different lattice spacings.[21]-[24] Experimental interest in antiferromagnetic $\gamma-\mathrm{Fe}$ precipitates in Cu-based alloys has been longstanding.[25] More recently the magnetic properties of epitaxial $\gamma-\mathrm{Fe}$ grown on $\mathrm{Cu}(100)$ were the subject of conflicting reports of para- and ferromagnetism, based on the interpretation of different angle-resolved photoemission experiments.[26],[27] The results of the present work are summarized in Figs. 6 and 7. A ferromagnetic hysteresis loop [see $\mathrm{Fig}, 6(\mathrm{a})$ ] was only observed for $\gamma-\mathrm{Fe} / \mathrm{Cu}(100)$ grown and measured at elevated temperature (e.g., $150^{\circ}$ or $190^{\circ} \mathrm{C}$ ). The ferromagnetism is metastable and disappears with time $(\sim 1 \mathrm{hr} \cdot)$ independent of the trace-impurity concentration detected in the Auger spectra.[11],[28] The ferromagnetism also disappears abruptly on cooling, and does not reappear on subsequent heating, as shown in Fig. 6(b) and (c). This is indicative of a first-order phase transition to the paramagnetic state, as suggested In Moruzzi's work on bulk $\gamma-\mathrm{Fe}$.[21]

Ferromagnetism can be restored by deposition, at elevated temperature, of a fresh layer of $\mathrm{Fe}$ on the existing film. Another striking aspect of the $\gamma-\mathrm{Fe} / \mathrm{Cu}(100)$ ferromagnetism, shown in Fig. 7 , is that the Kerr intensity is constant, independent of $\mathrm{Fe}$ dosage, and corresponds to a monolayertype signal, based on the $\mathrm{Fe} / \mathrm{Au}(100)$ measurements. It is concluded that the ferromagnetism resides in the top layer, rather than the interfacial layer, since a fresh deposition at elevated temperature can restore the ferromagnetic response, as mentioned above.

Thus, $\gamma-\mathrm{Fe} / \mathrm{Cu}(100)$ is no ordinary system. The main points that need to be explained in a successful model of 1 ts magnetic properties are:

a) the ferromagnetism, with in-plane anisotropy, is observed only at elevated temperatures,

b) the ferromagnetism is metastable, and

c) the ferromagnetism is confined to the surface layer.

Numerous models can be considered. One possibility involves the interlayer surface relaxation having two closely lying minima, with the expanded one being associated with ferromagnetism, while the more stable contracted one is not. Support for this model would require quantitative LEED analysis[29] of the surface interlayer spacing values in the two different states, or spinpolarized total-energy calculations as a function of top-layer spacing. This model leaves open the question of why the ferromagnetic state cannot be stabilized for room-temperature depositions. Perhaps increased roughness, as discussed in subsection 3B, would obliterate the proposed ferromagnetic local minimum. Alternate models can readily explain some, but not all, of the main points 1isted above. For instance, a monolayer-level signal could be due to the under- 
lying Fe coupling in antiferromagnetic sheets to the enhanced-moment ferromagnetism of the top layer, as has been suggested in recent $\gamma$-Fe/Cu(100) spin-polarized calculations.[20],[30] $\mathrm{A}$ modeI that addresses the disappearance of the signal on cooling is that the anisotropy changes from being in-plane at elevated temperatures to perpendicular on cooling. [31], [32] The geometry of the present experiment is sensitive only to the in-plane magnetization component. The above discussion points out that there are many interesting avenues left to explore in the $\gamma-F e / C u(100)$ system in the future.

\section{SUMMARY}

Examples were chosen to highlight the value of simple magneto-optical experiments in impacting the exciting realm of ultra-thin, epitaxial magnetic films. There is an abundance of opportunities and challenges to face in the future. Once the first-generation experiments are complete, and the existence of ferromagnetism is documented in a representative sampling of mode1 systems, the obvious 1 imitation of the present approach will become apparent - that quantitative comparisons of the magnitude of the magnetic moment to theory cannot readily be made because magneto-optometry is not magnetometry. With this in mind, the present approach will still provide a convenient auxiliary characterization tool for next-generation studies.

\section{ACKNOWLEDGMENTS}

The work was supported by the U.S. DOE-BES Materials Sciences under contract W-31-109-ENG38. We acknowledge fruitful interactions with P. Grünberg, P. A. Montano, B. R. Cooper, A. J. Freeman, I. K. Schuller, and G. Zajac.

\section{REFERENCES}

1. S. D. Bader, E. R. Moog, and P. Grünberg: J. Magn. Magn. Mater. 53 (1986) L295; and in "Magnetic Properties of Low Dimensional Systems," Ed. L. M. Falicov and J. L. Morán-López, Springer-Verlag, Berlin (1986) p. 70.

2. E. R. Moog and S. D. Bader: Superlattices and Microstructures 1 (1985) 543.

3. G. A. Prinz: Phys Rev. Lett. 54 (1985) 1051.

4. C. L. Fu, A. J. Freeman, and T. Oguchi: Phys Rev. Lett. 54 (1985) 2700.

5. G. Zajac, S. D. Bader, and R. J. Friddle: Phys. Rev. B31 (1985) 4947.

6. K. Sato: Japanese J. App1. Phys. 20 (1981) 2403.

7. K. Binder and P. C. Hohenberg: IEEE Trans. Magn. 12 (1976) 66.

8. N. D. Mermin and H. Wagner: Phys. Rev. Lett. 17 (1966) 1133.

9. B. T. Jonker, K.-H. Walker, E. Kisker, G. A. Prinz, and C. Carbone: Phys. Rev. Lett. 57 (1986) 142.

10. J. G. Gay and R. Richter: Phys. Rev. Lett. 56 (1986) 2728.

11. S. D. Bader and E. R. Moog: J. App1. Phys. (1987) in press; and TMS Proc. (1987) submitted.

12. R. H. Victora and L. M. Falicov: Phys. Rev. B31, (1985) 7335 .

13. G. Allan: Surf. Sci. 74 (1978) 79; Phys. Rev. B19 (1979) 4774.

14. C. Rau and S. Eichner: Phys. Rev. Lett. 47 (1981) 939; J. Magn. Magn. Mater. 30 (1982) 141; $31-34$ (1983) $874 ; 35$ (1983) 25.

15. F. Meier, D. Pescia, and T. Schriber: Phys. Rev. Lett. 48 (1982) 645.

16. L. E. Klebanoff, S. W. Robey, G. Liu, and D. A. Shirley: Phys. Rev. B30 (1984) 1048; 31 (1985) 6379; J. Magn. Magn. Mater. 54-47 (1986) 728.

17. E. R. Moog, S. D. Bader, P. A. Montano, G. Zajac, and T. H. Fleisch: Superlattices and Microstructures (1987) in press.

18. S. D. Bader, G. Zajac, A. J. Arko, M. B. Brodsky, T. I. Morrison, N. Zaluzec, J. Zak, R. L. Benbow, and Z. Hurych: Phys. Rev. B33 (1986) 3636.

19. D. R. Grempel: Phys. Rev. B24 (1981) 3928.

20. A. J. Freeman: private communication.

21. V. L. Moruzz1: Phys. Rev. Lett, 57 (1986) 2211.

22. V. L. Moruzz1, P. M. Marcus, K. Schwartz, and P. Mohn: Phys. Rev. B34 (1986) 1784.

23. F. J. Pinski, J. Staunton, B. L. Gyorffy, D. D. Johnson, and G. M. Stocks: Phys. Rev. Lett. 56 (1986) 2096.

24. C. S. Wang, B. M. Kleln, and H. Krakauer: Phys. Rev. Lett. 54 (1985) 1852.

25. U. Gonser, C. J. Meechan, A. H. Muir, and H. Wledersich: J. Appl. Phys. 34 (1963) 2373.

26. A. Amiri Hezaveh, G. Jennings, D. Pescia, R. F. Willis, K. Prince, M. Surman, and A. Bradshaw: Solid State Commun. 57 (1986) 329.

27. M. F. Onellion, C. L. Fu, M. A. Thompson, J. L. Erskine, and A. J. Freeman: Phys. Rev. B33, (1986) 7322 .

28. G. W. Fernando, Y. C. Lee, P. A. Montano, B. R. Cooper, E. R. Moog, H. M. Natk, and S. D. Bader: J. Vac. Sci. Technol. (1987) in press.

29. Y. Daric1, J. Marcano, H. Min, and P. A. Montano: Surf. Sci. (1987) in press.

30. B. R. Cooper: private communication.

31. W. Kümmerle and U. Gradmann: Phys. Stat. Sol A45 (1978) 171.

32. U. Gradmann: J. Magn. Magn. Mater. 54-57 (1986) 733. 